\section{Setting the trend with sportswear technology}

Leading workwear provider, Grahame Gardner Ltd, has unveiled an innovative new range of garments for healthcare professionals, bringing uniform design together with the latest in sportswear technology in a UK workwear first.

The Leicester-based firm has launched its 'Advance' collection in response to increasing demand from businesses for more customisable and contemporary uniforms that provide increased comfort and resilience.

The range, which consists of six styles, uses a unique new fabric called 'Aura' which, like sportswear fabrics, provides the wearer with moisture control as well as durability.

Customers can choose from two products, 'Cut and Sew' - which offers a range of 16 colours and trim options, or 'Advance Dye Sublimation' - which goes a step further allowing any colour (including pantone matching), any image or pattern, or even a company logo to create bespoke panels and trims. This option is the latest advance in permanent printing as the ink actually impregnates the fabric so is unaffected by washing or ironing.

Gary Lory, Managing Director of Grahame Gardner, said: 'We're thrilled to launch our new Advance range after 18 months of research and development, drawing on our extensive experience within both the workwear sector and the sportswear expertise of our sister brand GFORCE Teamwear.

'We've seen a significant increase in the number of customers looking for more bespoke workwear solutions, who understand

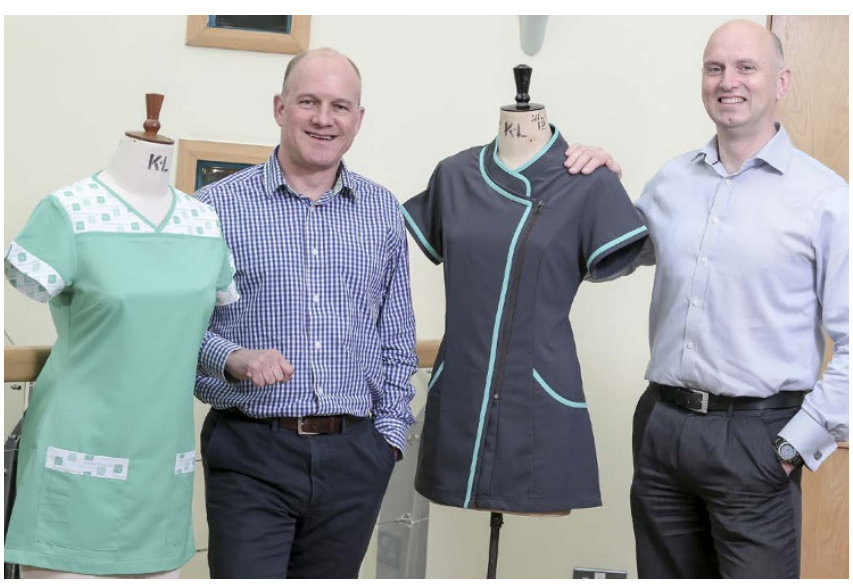

the importance of building and maintaining a strong brand that represents their organisation to both the people who utilise their services and their staff.

'The state-of-the-art Aura fabric combined with the latest dye-sublimation printing techniques provide a range that's not only functional and well-designed, but that works hard for the wearer too, using sportswear technology and techniques to create a more durable and performance-focused garment.'

Due to the techno-fibre of the Aura fabric, Grahame Gardner's new Advance range can be washed by hand or in a washing machine at 60 degrees, and doesn't need to be ironed.

For further information, visit www.grahamegardner.co.uk or call 01162556326.

\title{
Amalgam separators a must-have
}

Amalgam separators are a must for all dental practices, ensuring safe and effective separation of amalgam and compliance with the various hazardous waste regulations. As such, the separators must be fitted so as to protect all possible routes by which amalgam could enter the drains, including dirty sinks where instruments that have come into contact with amalgam are washed.

The PureMotion amalgam separator from Initial Medical is proven to be highly effective, removing up to $99.8 \%$ of amalgam particulate from waste water. BS ISO EN 111:43 approved - a requirement of all amalgam separators in the UK - it is suitable for all types of suction systems and can be retrofitted to existing pumps with no hassle.

What's more, with easy installation, no maintenance and minimal surgery down time, it really is a trouble-free solution for every dental practice.

\section{Initial}

The Experts in Healthcare Waste Management

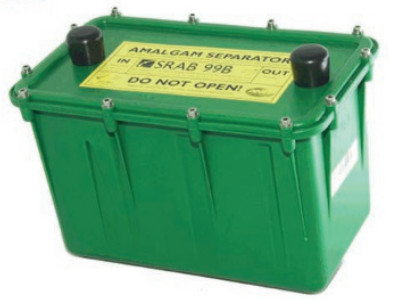

To help protect your staff, your patients and the environment, make sure your amalgam waste disposal protocols are up to scratch. Find out how the PureMotion amalgam separator from Initial Medical could improve your compliance today.

For further information visit www. inital.co.uk/medical or call 08708504045 .

\section{This glass is half-full}

With a higher surface hardness than many comparable materials, ${ }^{1}$ the all new Ketac Universal Aplicap glass ionomer restorative from $3 \mathrm{M}$ Oral Care is a reliable choice for stress-bearing restorations. ${ }^{2}$

Easy to use, ${ }^{3}$ strong and fast, ${ }^{4}$ this restorative material has no need for conditioner, coating ${ }^{5}$ or layering ${ }^{6}$ - meaning you can enjoy a far simpler workflow for a wide range of restorative indications. ${ }^{7}$

In fact, since it requires just four steps, ${ }^{8} \mathrm{Ketac}$ Universal Aplicap restorative helps reduce chair time - perfect for restless, caries-prone patients. ${ }^{4}$

To find out more about how Ketac Universal Aplicap is changing the way practitioners treat caries-prone patients, contact the team today. For more information, call 08456025094 or visit www.3Mespe.co.uk.

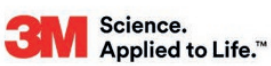

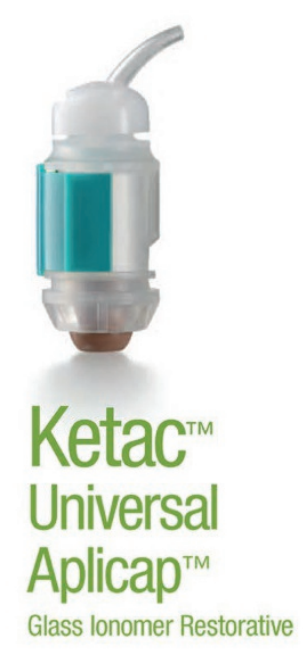

$3 M$, Ketac and Aplicap are trademarks of the $3 M$ Company.

1. $3 \mathrm{M}$ Oral Care Internal Data: Surface hardness is higher than several competitive glass ionomer restoratives; 2015. Claim no. 6259

2. $3 \mathrm{M}$ Oral Care Internal Data: Ketac is a reliable choice for both non stress-bearing and stress-bearing restorations; 2014. Claim no. 6185

3. 3M Oral Care Internal Data: 50 out of 51 dentists believe dispensing of Ketac is easy; 2014. Claim no. 6224

4. 3M Oral Care Internal Data: Ketac I reduces chair time; 2015. Claim no. 6332

5. 3M Oral Care Internal Data: Ketac has no need for conditioner or coating; 2015. Claim no. 6331

6. 3M Oral Care Internal Data: Bulk Fill: no need for layering; 2014. Claim no. 6156

7. 3M Oral Care Internal Data: Fewer steps = simple; 2015. Claim no. 6333

8. 3M Oral Care Internal Data: Ketac requires fewer clinical steps; 2015. Claim no. 6326 